\title{
A RARE CASE OF BUPHTHALMOS AND BILATERAL NUCLEAR CATARACTS IN A NEONATE WITH CONGENITAL RUBELLA SYNDROME
}

Uppin Narayan Reddy ${ }^{1}$, Swathi Chacham ${ }^{2}$, Jillalla Narsing Rao ${ }^{3}$, A. Vamshee Priya ${ }^{4}$, Afreen Mahmood ${ }^{5}$, Jakkampudi Nagasravani ${ }^{6}$, Ahmedullah Khan ${ }^{7}$

\section{HOW TO CITE THIS ARTICLE:}

Uppin Narayan Reddy, Swathi Chacham, Jillalla Narsing Rao, A. Vamshee Priya, Afreen Mahmood, Jakkampudi Nagasravani, Ahmedullah Khan. "A Rare Case of Buphthalmos and Bilateral Nuclear Cataracts in a Neonate with Congenital Rubella Syndrome". Journal of Evolution of Medical and Dental Sciences 2014; Vol. 3, Issue 25, June 23; Page: 6965-6969, DOI: 10.14260/jemds/2014/2847

ABSTRACT: BACKGROUND: Congenital rubella syndrome (CRS) is the second leading cause of nontraumatic childhood cataracts in India. While nuclear cataract is the most common abnormality reported in CRS, congenital glaucoma is a rarer manifestation. CASE REPORT: A 34weeks low birth weight, male neonate was born by vaginal delivery with normal APGARS. The neonate had sparse hypo pigmented hair over the scalp, along with hypopigmented eye brows and eye lashes. There were erythematous lesions over palms, soles and groin region. Eye examination revealed bilateral nuclear cataracts along with buphthalmos. The neonate also had clinical manifestations of PDA, which was confirmed by 2-D Echo. Systemic involvement was seen as hepatosplenomegaly and bilateral cryptorchidism. Hence CRS was suspected and further evaluation was done. There was thrombocytopenia, mild unconjugated hyperbilirubinemia with elevated transaminases. Neurosonogram was normal and there were no intra cranial calcifications. TORCH profile in both mother and baby showed elevated rubella IgM levels confirming CRS. The neonate received supportive and symptomatic treatment. DISCUSSION: congenital nuclear cataracts are reported in $60-80 \%$ of CRS, while buphthalmos is rarely seen, more so in neonatal period. PDA occurs in $50 \%$ of CRS and two-thirds have hepatosplenomegaly. Rubelliform rash is infrequent in neonates with CRS. CONCLUSION: We report a preterm low birth weight, male neonate with congenital rubella syndrome and its rare manifestations.

KEYWORDS: Congenital rubella syndrome, congenital buphthalmos, TORCH infection, congenital cataract, congenital heart disease.

ABBREVIATIONS: CHD, CRS, PDA, TORCH.

INTRODUCTION: Congenital Rubella syndrome (CRS) results from in utero transmission of rubella virus during maternal primary infection. Rubella was initially described as a benign contagious disease by Veale, a Scottish physician in $1866 .{ }^{1}$ The classical triad of congenital rubella syndrome was first described by Sir Norman Gregg, which consists of congenital cataracts, deafness and heart defects. ${ }^{2}$ Maternal infection has a spectrum of manifestations ranging from asymptomatic viremia to miscarriage, still births and severe birth defects in infants.

Viremia before 11 weeks of gestation leads to both congenital heart defects and deafness, while viremia between 13-16 weeks leads to deafness only. As with other TORCH infections, antenatal infection after 16 weeks of gestation rarely leads to developmental anomalies. ${ }^{3}$ In utero infection within 16weeks of gestation shares common pathology. Though the exact teratogenic mechanism of in utero rubella infection is not known, viral replication in cell clones during fetal organogenesis might be responsible. ${ }^{4}$ While cataracts and microphthalmia occur in $2 / 3$ of cases, 
congenital glaucoma is rare, more so in the neonate..$^{5}$ Sensory neural or central deafness is the most common sequel, manifesting in $80 \%$ of infected children. ${ }^{6}$

CASE REPORT: An out born, 34weeks, low birth weight (birth weight-1.7kgs) male neonate, born to a second gravida by spontaneous vaginal delivery with normal APGARs. Maternal TORCH profile showed elevated rubella IgM levels. The neonate was admitted soon after birth with respiratory distress, requiring supplemental oxygen. Examination revealed sparse hypo-pigmented hair over the scalp with scanty eye brows and eye lashes. Erythematous lesions were seen over palms, soles and groin region (as shown in fig-1A\&1B).

Buphthalmos (congenital glaucoma with corneal clouding) and bilateral nuclear cataracts were also seen (as shown in fig-2, C\&D). Systemic examination revealed pre-cardial activity, pansystolic murmur (suggestive of congenital heart defect-PDA) with hepatosplenomegaly and bilateral cryptorchidism. Clinically, congenital rubella syndrome (CRS) was diagnosed and the neonate was further worked up.

Sepsis screen was positive along with thrombocytopenia, unconjugated hyperbilirubinemia and elevated transaminases. Chest radiograph showed cardiomegaly and 2D Echo revealed a moderate to large PDA (2mm) with left to right shunt. Neurosonogram was normal and there were no intra-cranial calcifications in skull radiograph. Elevated rubella IgM levels were seen in the neonate, confirming CRS. The neonate received supportive and symptomatic treatment. It was discharged on decongestive treatment for PDA.

DISCUSSION: Chronic infection of the fetus with rubella virus leads to progressive damage which manifests as congenital rubella syndrome (CRS). With widespread immunization against rubella, the incidence of fetal exposure to rubella and CRS has drastically decreased, although not completely eradicated. It leads to a wide spectrum of manifestations ranging from acute manifestations in the neonatal period to progressive psychomotor retardation and deafness manifesting after infancy. ${ }^{6}$

The severity of damage depends on virulence of the organism and the gestational period of exposure. 6 The earlier the exposure, the worse is the outcome. The earlier the exposure, the higher will be the frequency of defects of CRS (more than $80 \%$ in the first 12 weeks of pregnancy, 54\% at 1314 weeks, and $25 \%$ at the end of second trimester). Risk of fetal transmission of rubella is highest if, the mother develops infection within first 12 weeks of gestation and decreases in the second trimester.

This transmission rate increases again in the last 10 weeks of gestation with $100 \%$ transmission at 36 weeks and beyond. Changes in the placental structure with increased resistance in the second trimester and later thinning of the trophoblast permitting late gestation passage. With increasing gestation, the incidence of ocular and cardiac defects declines while that of hearing defects increases. In the early pregnancy transplacentally acquired virus gets disseminated in the rapidly growing yet immune-deficient Fetus. ${ }^{6}$ This leads to impairment of the organogenesis and severe organ defects.

CRS often leads to prematurity and intra uterine growth retardation which manifested in the index case. Amongst the various congenital defects reported, central hearing loss is the commonest one. ${ }^{6}$ Interestingly severe neural hearing loss is more common than milder forms. Its presence alone can denote CRS. ${ }^{6}$ 
Nuclear cataract is the commonest ocular finding while congenital glaucoma is rarely seen. Apparent rarity of combined glaucoma and cataracts in a neonate with CRS has been reported by many studies..$^{7-9}$ The nuclear cataract results from viral invasion of the developing lens. Congenital glaucoma results from either failure of absorption of mesoderm at the angle of anterior chamber or from failure of differentiation of canal of Schlemm. The increased intraocular pressure leads to corneal clouding. The incidence of glaucoma in congenital rubella syndrome varies from 10-25\% from various studies. ${ }^{6,8-10}$

The index case had this rare combination of cataracts and glaucoma. Often it is seen at a later age than in the neonatal period, which makes its presence unique in the index case. Other ocular abnormalities like microphthalmia and salt pepper retinopathy can also manifest in CRS. Characteristic cardiac defects that occur in CRS include patent ductus arteriosus (PDA), pulmonary stenosis and ventricular septal defect (VSD). Cardiac lesions are more frequently prevalent in neonates with ocular lesions. ${ }^{6}$ Index case had PDA along with ocular manifestations.

Other infrequent manifestations include hepatosplenomegaly, meningo-encephalitis, thrombocytopenia, rubelliform rash, bone lucencies, cryptorchidism and diabetes mellitus. Hepatosplenomegaly with elevated transaminases, bilateral cryptorchidism, rubelliform rash and thrombocytopenia were present in the index neonate. Microcephaly with mental retardation, anemia, hypogammaglobulinemia, thymic hypoplasia, thyroid abnormalities and polycystic kidney disease were also reported in CRS.

Onset of some of the abnormalities of congenital rubella may be delayed by months to years. Along with CRS, maternal infection near term can lead to neonatal rubella due to transplacental viremia coupled with insufficient maternal antibodies. Passive immunization has a doubtful role in preventing progressive disease. ${ }^{11}$

Post natal diagnosis of suspected cases is by isolating rubella virus from urine or oropharynx, detecting rubella specific IgM in cord or neonatal blood and by finding elevated rubella IgG titers over time. ${ }^{12}$ No specific treatment exists for congenital rubella. The best mode of prevention is by immunizing all susceptible adults. If a woman receives rubella vaccine, conception should be delayed till 3months after vaccination to avoid the risk of fetal infection.

CONCLUSION: Congenital rubella syndrome manifesting in a preterm, low birth weight male neonate with rare combination of congenital nuclear cataracts, congenital glaucoma and rubelliform rash. Presence of many other infrequent, systemic manifestations with positive serology makes this case unique.

\section{REFERENCES:}

1. Veale H.history of an epidemic of Rothelin with observations on its pathology. Edin Med J 1866; 12: 404-414.

2. Gregg NM.Congenital cataract following German measles in the mother.Trans Ophthalmol Soc Aust 1941;3:35-46.

3. Jenkins KJ, Correa A, Feinstein JA, et al. Non-inherited risk factors and congenital cardiovascular defects: Current knowledge: a scientific statement from the American Heart Association Council on Cardiovascular Disease in the Young: endorsed by the American Academy of Pediatrics. Circulation 2007; 115(23): 2995-3014. 
4. De Oliveira SA, Camacho LA, De Medeiros Pereira AC, et al. Performance of rubella suspect case definition: implications for surveillance. Rev Saude Publica. 2006; 40:450-6.

5. Robinson JL, Lee BE, Preiksaitis JK, et al. Prevention of congenital rubella syndrome-what makes sense in 200? Epidemiol Rev.2006; 28:81-87.

6. Kerry T Givens, David A Lee, Thomas Jones, Duane M Ilstrup. Congenital rubella syndrome: Ophthalmic manifestations and associated systemic disorders. Br J Ophthalmology.1993; 77:358-363.

7. Boger WPIII.Late ocular complications in congenital rubella syndrome. Ophthalmology. 1980;87:1244-1252.

8. Sever JL, South MA, Shaver KA. Delayed manifestations of congenital rubella. Rev Infect Dis.1985; 7(suppl): S164-169.

9. Richard JM, Friendly DS.Ocular findings in Pediatric systemic disease.Pediatr Clin North Am 1983;30:1123-1144.

10. P Vijayalakshmi, T Amala Rajasundari, Noela Marie Prasad, et al., Prevalence of eye signs in congenital rubella syndrome in South India: A role for population screening. Br J Ophthalmol 2007; 91:1467-1470.

11. Wong LM, Medrano J. Real-Time PCR for mRNA quantification. Bio- Techniques.2005; 39: 75-85.

12. Surveillance Guidelines for Measles, Rubella and Congenital Rubella Syndrome in the WHO European Region, World Health Organization. 2009; 1-48.

Fig. 1: Denotes rubelliform rash over sole (A) and over the groin region (B).

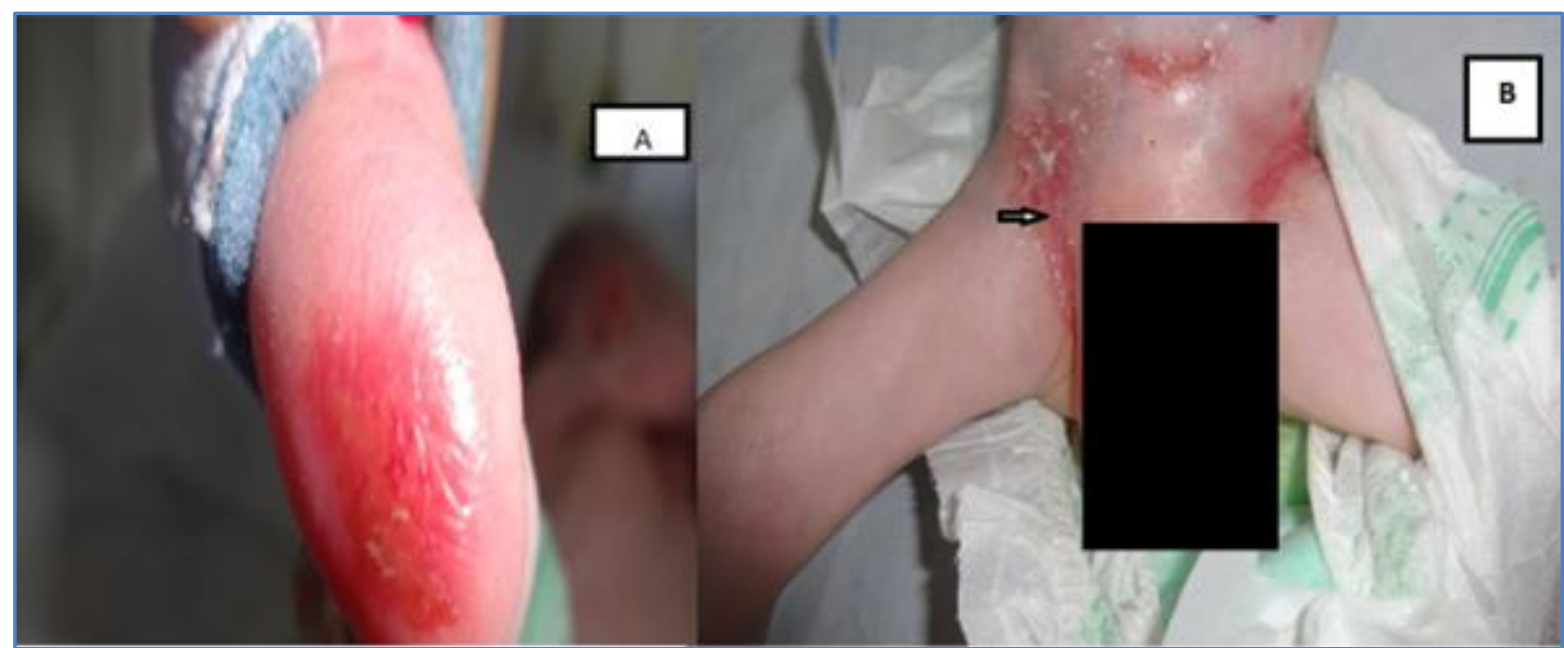

Figure 1A

Figure 1B 


\section{CASE REPORT}

Fig. 2: Denotes corneal opacity (C) and nuclear cataract (D).

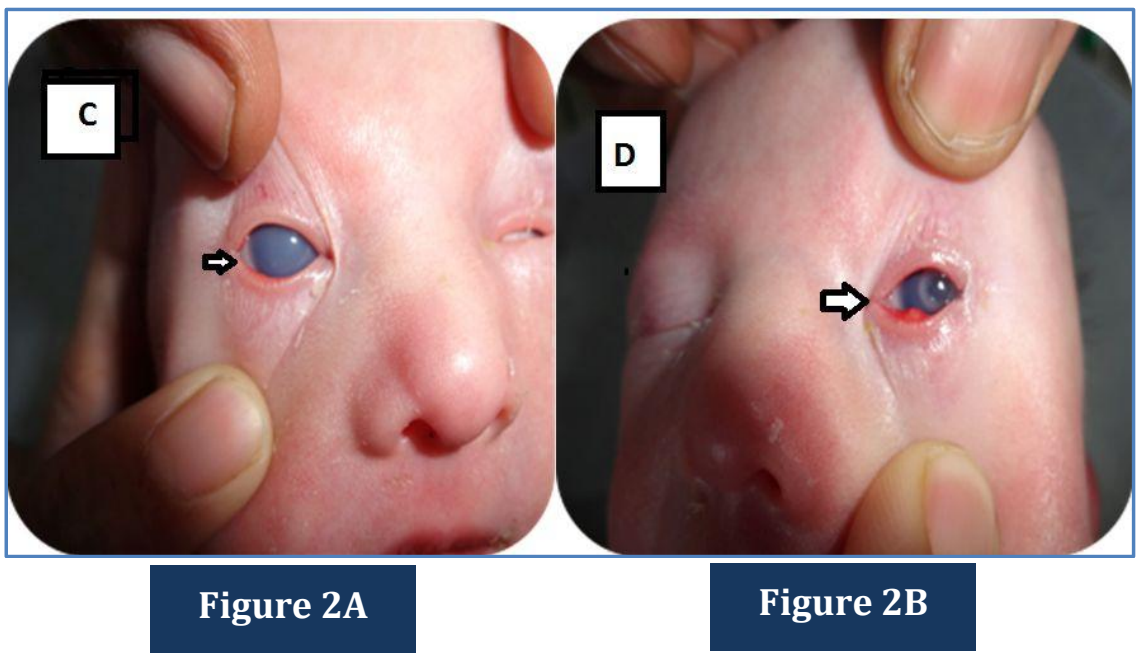

\section{AUTHORS:}

1. Uppin Narayan Reddy

2. Swathi Chacham

3. Jillalla Narsing Rao

4. A. Vamshee Priya

5. Afreen Mahmood

6. Jakkampudi Nagasravani

7. Ahmedullah Khan

\section{PARTICULARS OF CONTRIBUTORS:}

1. Professor and HOD, Department of Pediatrics, Princess Esra Hospital, Deccan College of Medical Sciences, Hyderabad, Andhra Pradesh, India.

2. Assistant Professor, Department of Neonatology, PGIMER, Chandigarh.

3. Professor, Department of Paediatrics, Princess Esra Hospital, Deccan College of Medical Sciences, Hyderabad, Telangana, Sha-Ali Banda.

4. Senior Resident, Department of Paediatrics, Princess Esra Hospital, Deccan College of Medical Sciences, Hyderabad, Telangana, ShaAli Banda.

5. Junior Resident, Department of Paediatrics, Princess Esra Hospital, Deccan College of Medical Sciences, Hyderabad, Telangana, ShaAli Banda.
6. Junior Resident, Department of Paediatrics, Princess Esra Hospital, Deccan College of Medical Sciences, Hyderabad, Telangana, ShaAli Banda.

7. Junior Resident, Department of Paediatrics, Princess Esra Hospital, Deccan College of Medical Sciences, Hyderabad, Telangana, ShaAli Banda.

\section{NAME ADDRESS EMAIL ID OF THE CORRESPONDING AUTHOR:}

Dr. Swathi Chacham,

Princess Esra Hospital,

Deccan College of Medical Sciences, Hyderabad, Andhra Pradesh, India.

Email: swathi.m.lahiri@gmail.com

Date of Submission: 03/06/2014. Date of Peer Review: 04/06/2014. Date of Acceptance: 10/06/2014. Date of Publishing: 20/06/2014. 